\title{
A Clinical Epidemiological Analysis of Prognostic Nutritional Index Associated with Diabetic Retinopathy
}

This article was published in the following Dove Press journal: Diabetes, Metabolic Syndrome and Obesity: Targets and Therapy

\author{
Lijuan Yang \\ Weihui Yu \\ Wei Pan \\ Shuoping Chen \\ Xiwen Ye \\ Xuejiang Gu \\ Xiang $\mathrm{Hu}$
}

Department of Endocrine and Metabolic Diseases, The First Affiliated Hospital of Wenzhou Medical University, Wenzhou, 325000, Zhejiang Province, People's Republic of China
Correspondence: Xiang Hu; Xuejiang Gu Department of Endocrine and Metabolic Diseases, The First Affiliated Hospital of Wenzhou Medical University, Ouhai District, Wenzhou, 325000, Zhejiang Province, People's Republic of China Tel +86-577-5557938I

Email huxiang@wmu.edu.cn; guxuejiang@wmu.edu.cn
Purpose: Prognostic nutritional index (PNI) is an effective tool to evaluate the nutritional conditions and predict prognosis, but clinical data are limited for the use of PNI in diabetic retinopathy (DR). This study aimed to investigate the relationship of PNI with the prevalence and severity of DR in patients with type 2 diabetes mellitus (T2DM).

Patients and Methods: This cross-sectional analysis enrolled 1023 individuals with T2DM hospitalized between 2017-2020. PNI was calculated as $10 \times$ serum albumin $(\mathrm{g} / \mathrm{l})$ $+0.005 \times$ total lymphocyte count (cells $/ \mathrm{mL}$ ). DR severity was categorized as no, nonproliferative, and vision-threatened DR (VTDR) according to the modified Airlie House classification. Multivariate-adjusted odds ratio (OR) with $95 \%$ confidence interval (CI) for the prevalent DR in the top (Q4) compared with the bottom quartile (Q1) of PNI levels were estimated by using logistic regression analyses.

Results: PNI levels were significantly lower in individuals with VTDR than those with no and nonproliferative DR (both $P<0.001$ ), and the proportions of individuals with DR were significantly decreased in the top quartile compared with the bottom quartile of PNI levels $(P<0.001)$. After adjustments for age, gender, DM duration, obesity-related risk factors and clinical biochemical parameters, the higher levels of PNI were significantly associated with a lower prevalence of DR (Q4 vs Q1: OR $=0.402,95 \%$ CI: $0.250-0.649, P<0.001$ ), with a $5.9 \%$ reduction in the prevalence of DR for a per-unit increment in the levels of PNI (OR $=0.941,95 \%$ CI: $0.911-0.972$, $P<0.001$ ). The association of PNI and obesity-related indexes (body mass index and waist circumference) with the severity of DR was independent of each other $(\mathrm{P}<0.001)$.

Conclusion: PNI was inversely and independently associated with the severity and prevalence of DR, which suggested that PNI could likely be used to predict DR prognosis in clinical practice.

Keywords: diabetic retinopathy, type 2 diabetes mellitus, prognostic nutritional index, obesity

\section{Introduction}

Diabetic retinopathy (DR), as the most unique microvascular complication of diabetes, is present in over one-third of diabetic population globally. ${ }^{1}$ DR is a primary reason for vision impairment and blindness, and its severity threatens the health burden on society. ${ }^{2}$ The traditional concept holds that dyslipidaemia, hypertension, hypercholesterolaemia, and anaemia are the factors consistently associated with the development of retinopathy. ${ }^{1,3,4}$ Apart from those factors, accumulating evidence has demonstrated that malnutrition is a potential DR risk factor. ${ }^{5}$ Malnourished diabetic patients tend to have diabetes-associated diseases including 
DR, renal failure, infections, and mortality due to diabetic foot ulcer. ${ }^{6,7}$ However, to the best of our knowledge, nutrition status examinations are complex and subjective, and there is no validated assessment index of DR risk.

The aetiology of retinopathy is not fully understood; nevertheless, emerging evidence has shown that inflammatory processes play a key role in the presence and progression of $\mathrm{DR}^{8}$ A high prevalence of malnutrition is observably related to immunosuppression and T lymphocyte disorders in diabetic patients. ${ }^{9,10}$ The role of malnutrition in inflammation determines its critical role in the development of diabetic complications. T cells have been shown to play a crucial role in diabetic complications. Studies have revealed that the imbalance of Th1/ Th17/Treg cells in diabetic patients with complications might manifest as $\mathrm{CD} 4^{+} \mathrm{CD} 25^{\text {hi }}$ Tregs with decreased immune function and increased inflammatory Th1 and Th17 cells. $^{11,12}$ Serum albumin was recognized to be related to nutrition and inflammation, which was a source of thiols that could scavenge reactive oxygen. ${ }^{13} \mathrm{Zhu}$ et al discovered that serum albumin was strongly associated with DR and diabetic kidney disease (DKD) independent of sex, age, duration of diabetes, smoking status, obesityrelated indexes, clinical biochemical parameters, and other blood parameters in Chinese T2DM population, which suggested that diabetic chronic vascular complications caused by hypoalbuminemia attributed to chronic inflammation and oxidative stress. ${ }^{14}$ Based upon the above theory, we hypothesized that prognostic nutritional index (PNI) which is obtained from the serum albumin level and lymphocyte count could represent convenient and cost-effective biomarkers for assessing the immunenutrition status of diabetes patients and predicting the progression of DR.

Therefore, the primary purpose of this study was to discuss the association of PNI with the presence and severity of DR in Chinese population with T2DM.

\section{Patients and Methods}

\section{Study Population}

The present study recruited a total of 1023 inpatients with T2DM in the Department of Endocrine and Metabolic Disease in the First Affiliated Hospital of Wenzhou Medical University, from March 2017 to March 2020. For the candidate subjects, the exclusion criteria were described below: 1) age under 40 years old; 2) malignant tumor, infection, acute diabetic complications, autoimmune diseases, or blood diseases; 3) severe liver, heart, or kidney disease; 4) major trauma, surgery in the previous 6 months, or pregnancy; 5) cerebrovascular disease; 6) psychiatric disease; 7) current treatment with systemic corticosteroids; or 8) cataract, glaucoma or any other eye disease were excluded by the ophthalmologist.

The present study was conducted in compliance with the Declaration of Helsinki and preapproved by the Ethics Committee of The First Affiliated Hospital of Wenzhou Medical University. All subjects provided signed informed consent prior to participation.

\section{Anthropometric and Biochemical Measurements}

Every individual was requested to have a general physical examination by trained doctors. Anthropometrics including body height, weight, waist circumference (WC), and blood pressure (BP) were measured by standard methods. Body mass index (BMI) was calculated according to the following formula: $\mathrm{BMI}=$ weight $(\mathrm{kg}) /$ height ${ }^{2}\left(\mathrm{~m}^{2}\right)$. WC was assessed at the horizontal line between the lowest rib and the anterior superior iliac spine on the midpoint line. According to Expert Consensus on Integrated Management of Type 2 Diabetes Mellitus Complicated with Obesity in China, ${ }^{15}$ overall obesity was defined as BMI $\geq 24 \mathrm{~kg} / \mathrm{m}^{2}$, and abdominal obesity was defined in men and women as $\mathrm{WC} \geq 90 \mathrm{~cm}$ and $\mathrm{WC} \geq 85 \mathrm{~cm}$, respectively. Systolic BP (SBP) and diastolic BP (DBP) were taken three times with a mercury sphygmomanometer at a 3-min interval after taking a 10 -min break.

Blood was sampled from the antecubital vein between 6:00 and 10:00 am following 10-12 h of fasting. The laboratory tests included fasting plasma glucose (FPG), glycated haemoglobin ( $\mathrm{HbAlc})$, serum albumin, white blood cell (WBC) count, total lymphocyte count (Tlc) count, red blood cell (RBC) count, platelets (PLT), haemoglobin ( $\mathrm{Hb}$ ), serum lipid spectra (including total cholesterol [TC], lowdensity lipoprotein cholesterol [LDL-c], high-density lipoprotein cholesterol [HDL-c], triglycerides [TGs]), serum creatinine (Scr), and serum uric acid (SUA). All blood samples were assayed intensively within 24 hours.

\section{Fundus Examination}

Eye examinations were operated by a professionally trained endocrinologist nurse in the darkroom free of mydriasis with VISUCAM PRONM (VISUCAM 200, 
serial number: $1,098,263)$, which was used to capture $45^{\circ}$ $\mathrm{C}$ colour digital images of the binocular fundus. When a fundus film was not clear, mydriasis fundus examination or fundus fluorescein angiography needed to be performed. The severity of DR was determined according to the modified Airlie House classification, ${ }^{16}$ and the patients in the present study were divided into three groups as no DR, non-proliferative DR (NPDR), and vision-threatened DR (VTDR). Diagnosis of DR was performed by two ophthalmologists from the Eye Institute of the First Affiliated Hospital of Wenzhou Medical University blinded to the patients' clinical status. In case of different assessments of DR grades, a third ophthalmologist was consulted.

\section{Statistical Analysis}

Statistical analyses were carried out with SPSS version 16.0 (SPSS Inc., Chicago, IL, 167 USA). DR was analysed as bivariate (yes or no DR) and categorical variables: no DR, mild-to-moderate nonproliferative diabetic retinopathy (NPDR), and vision threatening diabetic retinopathy (VTDR). Baseline characteristics of individuals across three different DR severity groups were compared. Data are described as the mean \pm standard deviation or median with interquartile range for continuous variables, and described as numbers (percentages) for categorical variables. Categorical variables were compared by Chisquared test. Means were compared by one-way ANOVA, and medians were compared by KruskalWallis test. Univariate and multivariate logistic regressions were used to evaluate the association of factors including age, gender, DM duration, PNI, BMI, SBP, DBP, WC, HbAlc, Hb, RBC, WBC, PLT, Scr, SUA, TG, HDL-c, LDL-c and the presence of DR, which were assessed by univariate and multivariate logistic regressions. Logistic regression analyses were carried out to identify the relationship between PNI quartiles and the presence of DR. Variance analyses were performed to investigate the association between PNI and DR, as well as the influence of BMI and WC. The threshold of statistical significance was established at 0.05 for two-tailed $P$-values.

\section{Results \\ Clinical Characteristics of the Research Individuals}

A total of 1023 individuals with an average age of 59.16 \pm 5.95 years (age range: $50-75$ years) were admitted in the present study, involving 501 individuals with no DR, 365 individuals with NPDR, and 157 individuals with VTDR. PNI was significantly lower in individuals with severe DR than in individuals with no DR and NPDR (both $P<0.001)$. In addition, subjects with severe DR were more likely to be older, have a long duration of diabetes, have higher levels of FPG, SBP, and PLT, and have lower levels of albumin, Tlc, PNI, Hb, and RBC than subjects with no DR and NPDR (all $\mathrm{P}<0.001$, except $P=0.012$ for FPG). Other variables showed no significant difference among the three groups (all $\mathrm{P}>0.05$, Table 1 ).

\section{The Presence of DR Among PNI Quartiles}

The current study participants were further classified into four subgroups on the basis of PNI quartiles (PNI level: Q1: $\leq 44.80$, Q2: 44.85 48.35, Q3: 48.40 52.25, Q4: $\geq 52.30$ ). The distribution of no DR, and DR were stratified by the quartiles of PNI. There was a significant trend of a stepwise increase in no DR and a decrease in DR corresponding to an increase in PNI quartiles $(P<0.001)$. Adjusted for age and gender, the risks of DR were decreased by more than half in Q3 (odds ratio [OR] $=0.423, \quad 95 \% \quad \mathrm{CI}: 0.295-0.606, \quad P<0.001)$ and $\mathrm{Q} 4$ (OR=0.362, 95\% CI: $0.251-0.521, P<0.001)$ compared to Q1. Even after adjusting for age, gender, DM duration, BMI, WC, HbAlc, SBP, DBP, Hb, RBC, WBC, PLT, Scr, SUA, TG, HDL-c, and LDL-c, the decrease in risks of DR remained in $\mathrm{Q} 3 \quad(\mathrm{OR}=0.481,95 \% \mathrm{CI}: 0.308-0.751$, $P=0.001)$ and $\mathrm{Q} 4 \quad(\mathrm{OR}=0.402,95 \% \mathrm{CI}: 0.250-0.649$, $P<0.001)$ when compared to Q1. Moreover, the per unit increase in PNI was dramatically correlated with a $5.9 \%$ reduction in the prevalence of $\mathrm{DR}(\mathrm{OR}=0.941,95 \% \mathrm{CI}$ : 0.911-0.972, $P<0.001$ ) (Figure 1).

\section{Interaction of DR Between PNI and BMI, WC}

The current study participants were divided into high BMI group and low BMI group, high WC group and low WC group on the basis of obesity criteria. The results indicated that there were significant differences in PNI among those without DR to VTDR $(\mathrm{P}<0.001)$. The association of PNI and BMI with the severity of DR was independent of each other (Figure 2A). The association of PNI and WC with the severity of DR was independent of each other (Figure 2B). 
Table I Characteristics of Study Participants

\begin{tabular}{|c|c|c|c|}
\hline & No DR & NPDR & VTDR \\
\hline N & 501 & 365 & 157 \\
\hline Age(years) & $57.0(54.0-63.0)$ & $59.0(55.0-64.0)^{*}$ & $59.0(54.5-64.5)$ \\
\hline Female/Male & $199 / 302$ & $152 / 213$ & $68 / 69$ \\
\hline DM duration (months, 0-49I) & $68.0(15.0-136.0)$ & $134.0(84.0-188.0)^{* *}$ & $153.0(85.0-237.0)^{* *}$ \\
\hline Albumin $(g / l)$ & $39.46 \pm 3.58$ & $38.30 \pm 4.03 * *$ & $36.91 \pm 5.10 * * \neq$ \\
\hline Lymphocyte count (cells/mL) & $2.02 \pm 0.66$ & $1.94 \pm 0.63^{*}$ & $1.78 \pm 0.6 \mathrm{I}^{* * \dagger}$ \\
\hline $\mathrm{PNI}$ & $49.56 \pm 5.23$ & $47.95 \pm 5.27 * *$ & $45.75 \pm 6.30 * * \neq$ \\
\hline $\operatorname{BMI}\left(\mathrm{kg} / \mathrm{m}^{2}\right)$ & $24.61 \pm 3.07$ & $24.55 \pm 3.45$ & $24.26 \pm 3.15$ \\
\hline $\mathrm{DBP}(\mathrm{mmHg})$ & $73.51 \pm 10.12$ & $73.46 \pm 10.61$ & $73.89 \pm 11.70$ \\
\hline $\mathrm{SBP}(\mathrm{mmHg})$ & $126.36 \pm 17.27$ & $131.67 \pm 19.04 * *$ & $135.42 \pm 21.15^{* * \dagger}$ \\
\hline $\mathrm{WC}(\mathrm{cm}, 62.0-121.0)$ & $88.0(82.0-95.0)$ & $89.0(84.0-95.0)$ & $88.5(83.0-94.9)$ \\
\hline $\mathrm{HbAIc}(\%, 5.0-17.5)$ & $9.20(7.65-11.20)$ & $9.40(8.20-11.00)$ & $9.55(8.00-11.40)$ \\
\hline $\mathrm{FPG}(\mathrm{mmol} / \mathrm{L}, 3.9-23.7)$ & $7.00(5.70-8.80)$ & $7.30(5.80-9.45)$ & $8.05(5.70-10.28)^{*}$ \\
\hline $\mathrm{Hb}(g / L)$ & $|39.44 \pm| 3.8 \mid$ & $135.20 \pm 16.22 * *$ & $127.33 \pm 18.43^{* * \neq}$ \\
\hline RBC(cells/mL) & $4.58 \pm 0.45$ & $4.47 \pm 0.54^{*}$ & $4.28 \pm 0.67^{* * \ddagger}$ \\
\hline WBC(cells/mL) & $6.59 \pm 1.77$ & $6.61 \pm 1.76$ & $6.86 \pm 2.11$ \\
\hline PLT(cells/mL) & $215.00 \pm 60.08$ & $219.01 \pm 59.10$ & $230.97 \pm 85.59 *$ \\
\hline Scr(umol/L, 30.0-347.0) & $62.0(53.0-73.0)$ & $62.0(51.0-76.0)$ & $63.0(50.0-81.0)$ \\
\hline SUA(umol/L, I08.0-75I.0) & $302.0(244.8-263.5)$ & $307.5(256.0-367.0)$ & $322.0(269.0-391.0)^{*}$ \\
\hline $\mathrm{TG}(\mathrm{mmol} / \mathrm{L}, 0.5 \mathrm{I}-\mathrm{I0.46})$ & $1.45(1.05-2.05)$ & $1.49(1.04-2.27)$ & $1.55(1.12-2.21)$ \\
\hline LDL-c(mmol/L, 0.6I-5.58) & $2.48(1.95-3.18)$ & $2.43(1.87-3.12)$ & $2.55(1.90-3.38)$ \\
\hline $\mathrm{HDL}-\mathrm{c}(\mathrm{mmol} / \mathrm{L}, 0.48-3.13)$ & $1.00(0.85-1.20)$ & $\mathrm{I} .0 \mathrm{I}(0.85-1.2 \mathrm{I})$ & $1.02(0.84-1.20)$ \\
\hline
\end{tabular}

Notes: Data are the mean \pm standard deviation, median (interquartile range), and $\mathrm{n}(\%)$. $* \mathrm{P}<0.05$ versus No DR. $* * \mathrm{P}<0.00$ I versus No DR. ${ }^{\dagger} \mathrm{P}<0.05$ versus NPDR. ${ }^{\ddagger} \mathrm{P}<$ 0.00 I versus NPDR.

Abbreviations: DR, diabetic retinopathy; NPDR, nonproliferative diabetic retinopathy; VTDR, vision-threatening diabetic retinopathy; DM duration, diabetes mellitus duration; PNI, prognostic nutritional index; BMI, body mass index; DBP, diastolic blood pressure; SBP, systolic blood pressure; WC, waist circumference; HbAIc, glycated hemoglobin Alc; FBG, fasting blood glucose; Hb, hemoglobin; RBC, red blood cell; WBC, white blood cell; PLT, platelet; Scr, serum creatinine; SUA, serum uric acid; TG, triglyceride; LDL-c, low-density lipoprotein cholesterol; HDL-c, high-density lipoprotein cholesterol.

\section{Multivariate Analysis of the Risk of DR in Individuals with T2DM}

The risk factors of retinopathy analysis were conducted in individuals with T2DM. Results after multivariate analysis showed that PNI $(\mathrm{OR}=0.941, P<0.001)$, DM duration $(\mathrm{OR}=1.009, \quad P<0.001), \quad \mathrm{SBP} \quad(\mathrm{OR}=1.109, \quad P=0.001)$, HbAlc $(\mathrm{OR}=1.113, P=0.005), \mathrm{Hb}(\mathrm{OR}=0.975, P=0.008)$, and TG $(\mathrm{OR}=1.154, P=0.040)$ were independently correlated with DR (Table 2).

\section{Discussion}

The present study suggested that individuals with severe DR exhibited lower levels of PNI. The risk of DR decreased with an increase in PNI quartiles. The proportions of individuals with DR were significantly decreased in the top quartiles compared with the bottom quartile of PNI levels. After adjusting for age, gender, DM duration, obesity-related risk factors and clinical biochemical parameters, the level of PNI was associated with the prevalence of DR negatively and independently. Indicators of obesity, such as BMI and WC, could not interfere with the relationship between PNI and the severity of DR.

Some previous studies revealed that low socioeconomic status was inversely associated with DR incidence, while malnutrition might be one of the reasons behind the low socioeconomic status. ${ }^{17}$ Malnutrition is common in diabetes individuals, which impaired the clinical outcomes including a longer hospital stay, infection-related complication, mortality and so on. ${ }^{18}$ Previous research demonstrated that $14.1 \%$ of elderly diabetic patients suffer from malnourishment and the risk of malnutrition was up to 42.6\%. ${ }^{9}$ The reduction in immune cell number, particularly the $\mathrm{T}$ cells, resulted from malnutrition. In studies of mice fasted for $48 \mathrm{~h}$, thymocyte and splenocyte counts decreased, and $\mathrm{T}$ cell and $\mathrm{CD} 4+\mathrm{T}$ cell numbers from spleens from fasted mice were reduced by $40-50 \%$ compared to those from fed mice in control group. ${ }^{19,20}$ In human studies, children with malnutrition had atrophied primary lymphoid organs, decreased CD4+ and CD8+ T cells and leukopenia. ${ }^{21}$ Some cytokines and chemokines 


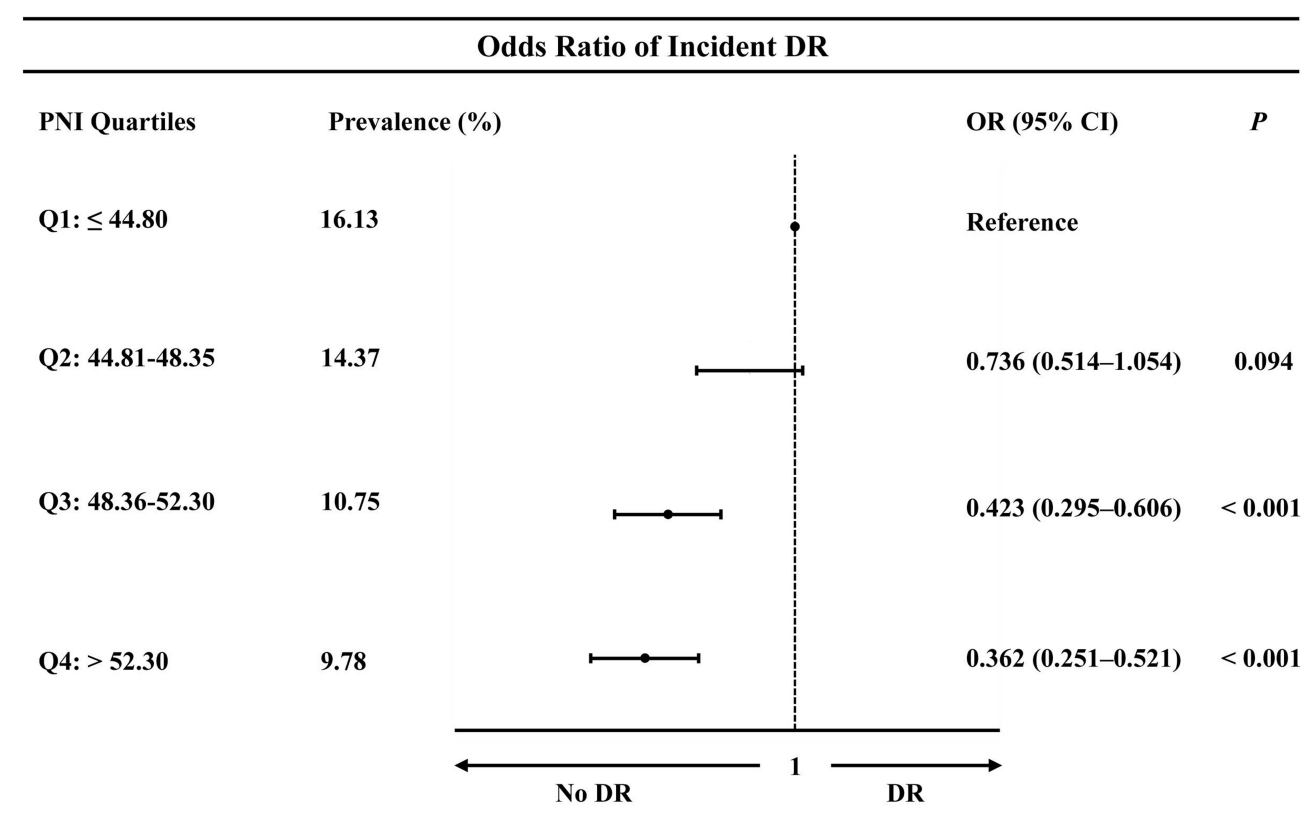

Figure I The presence of diabetic retinopathy among PNI quartiles.

A

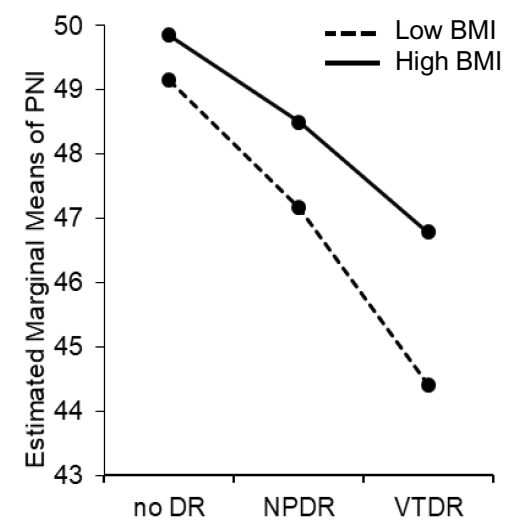

B

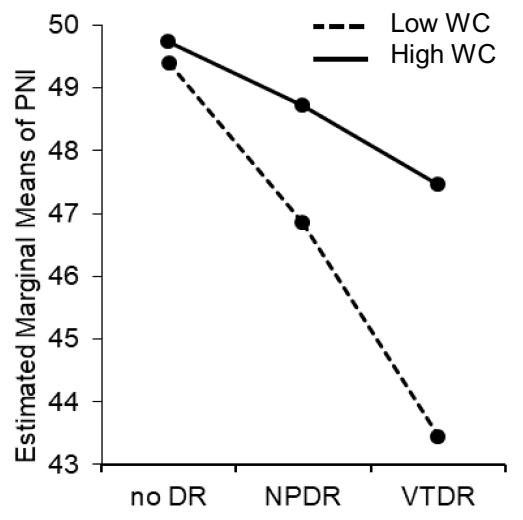

Figure 2 (A) Interaction of the present and severity of diabetic retinopathy between PNI and BMI. (B) Interaction of the present and severity of diabetic retinopathy between $\mathrm{PNI}$ and WC.

play a pivotal role in linking malnutrition and immunity, including a decline in leptin, interleukin-6 (IL-6), IL-8, macrophage migration inhibitor (MIF), and an increase in IL-10, IL-33, and mononuclear chemisorption factor-1 $(\mathrm{MCP}-1){ }^{22}$ In addition, it has been recognized that the levels of circulating inflammatory mediators are related to the presence and severity of DR. ${ }^{23}$ Therefore, DR might be related to malnutrition due to weakened immune defence, and inflammation contributes to DR development. In the present study, traditional risk factors for $\mathrm{DR}$, such as duration of diabetes, HbAlc, SBP, TG, and anaemia, were associated with DR. Furthermore, a nutrition indicator, PNI, stood out as an independent factor that was negatively correlated with DR, which challenged the above conventional risks of DR.

Buzby et al were the first to estimate the operative risk with PNI in gastrointestinal surgery. ${ }^{24}$ Subsequent researches on the predictive value of PNI for clinical outcome have also been addressed for malignancy, acute and chronic heart failure, peripartum cardiomyopathy, and systemic lupus erythematosus. ${ }^{25-29}$ As far as we are aware, existing data on the association between nutrition status and DR are sparse. Albumin, a clinical monitoring tool for nutritional assessment, was negatively associated with DR. ${ }^{14}$ Due to the influences contributed by liquid transfer, liver failure, metabolic and catabolic states, and infection, 
Table 2 Univariate and Multivariate Analyses of Factors Associated with DR in Patients with T2DM

\begin{tabular}{|c|c|c|c|c|}
\hline \multirow{3}{*}{$\begin{array}{l}\text { Variables } \\
\text { Age(years) }\end{array}$} & \multicolumn{2}{|c|}{ Univariate } & \multicolumn{2}{|c|}{ Multivariate } \\
\hline & \multicolumn{2}{|c|}{ P value $\mathrm{OR}(95 \% \mathrm{Cl})$} & \multicolumn{2}{|c|}{ P value $O R(95 \% \mathrm{Cl})$} \\
\hline & 0.012 & $1.027(1.006-1.048)$ & 0.395 & $0.988(0.961-1.016)$ \\
\hline Gender & 0.430 & $0.905(0.705-1.16 \mathrm{I})$ & 0.326 & $1.230(0.814-1.858)$ \\
\hline DM duration(years) & $<0.001$ & $1.009(1.007-1.010)$ & $<0.001$ & $1.009(1.007-1.011)$ \\
\hline $\mathrm{PNI}$ & $<0.001$ & $0.926(0.904-0.948)$ & $<0.001$ & $0.94 I(0.911-0.972)$ \\
\hline $\operatorname{BMI}\left(\mathrm{kg} / \mathrm{m}^{2}\right)$ & 0.473 & $0.986(0.949-1.025)$ & 0.071 & $0.932(0.864-1.006)$ \\
\hline $\mathrm{DBP}(\mathrm{mmHg})$ & 0.907 & $1.001(0.989-1.012)$ & 0.900 & $1.00 \mathrm{I}(0.98 \mathrm{I}-\mathrm{I} .002)$ \\
\hline $\mathrm{SBP}(\mathrm{mmHg})$ & $<0.001$ & $1.019(1.012-1.026)$ & 0.001 & $1.109(1.008-1.031)$ \\
\hline $\mathrm{WC}(\mathrm{cm})$ & 0.760 & $1.002(0.989-1.016)$ & 0.333 & $1.013(0.987-1.041)$ \\
\hline $\mathrm{HbAlc}(\%)$ & 0.221 & $1.035(0.980-1.093)$ & 0.005 & $1.113(1.033-1.199)$ \\
\hline $\mathrm{Hb}(\mathrm{g} / \mathrm{L})$ & $<0.001$ & $0.973(0.965-0.98 I)$ & 0.008 & $0.975(0.957-0.993)$ \\
\hline RBC(cells/mL) & $<0.001$ & $0.536(0.4 \mid 8-0.687)$ & 0.566 & $1.159(0.700-1.919)$ \\
\hline WBC(cells/mL) & 0.404 & $1.029(0.962-1.102)$ & 0.316 & $1.049(0.956-1.151)$ \\
\hline PLT(cells/mL) & 0.061 & $1.002(1.000-1.004)$ & 0.319 & $1.001(0.999-1.004)$ \\
\hline Scr (umol/L) & 0.002 & $1.009(1.003-1.015)$ & 0.294 & $1.005(0.996-1.014)$ \\
\hline SUA(umol/L) & 0.120 & $1.001(1.000-1.003)$ & 0.374 & $1.001(0.999-1.003)$ \\
\hline $\mathrm{TG}(\mathrm{mmol} / \mathrm{L})$ & 0.218 & $1.065(0.963-1.178)$ & 0.040 & $1.154(1.006-1.323)$ \\
\hline HDL-c(mmol/L) & 0.976 & $1.006(0.66 I-1.533)$ & 0.286 & I.356(0.775-2.374) \\
\hline LDL-c(mmol/L) & 0.645 & $0.968(0.844-1.110)$ & 0.998 & $1.000(0.838-1.194)$ \\
\hline
\end{tabular}

Abbreviations: DR, diabetic retinopathy; DM duration, diabetes mellitus duration; PNI, prognostic nutritional index; BMI, body mass index; DBP, diastolic blood pressure; SBP, systolic blood pressure; WC, waist circumference; HbAlc, glycated hemoglobin Alc; Hb, hemoglobin; RBC, red blood cell; WBC, white blood cell; PLT, platelet; Scr, serum creatinine; SUA, serum uric acid; TG, triglyceride; LDL-c, low-density lipoprotein cholesterol; HDL-c, high-density lipoprotein cholesterol.

which restrict the application of albumin in evaluating the nutrition status. Subjective Global Assessment (SGA) score was related to the presence and severity of $\mathrm{DR},{ }^{30,31}$ whereas, this nutritional screening tool needs to be evaluated subjectively by the experienced examiners. PNI was obtained from objective markers including albumin and lymphocyte count, reflecting nutrition and immunity. Therefore, the present study used PNI to assess nutrition status in diabetic patients with or without DR. The result suggested that DR was aggravated with the decrease in PNI.

BMI is another index to assess the nutritional condition in routine clinical practice. Previous studies revealed that low BMI caused by uncontrolled glycaemia was correlated with an increased risk of DR. ${ }^{32,33}$ Other studies discovered that high BMI or overnutrition, accompanied by hypertension and hyperlipidemia, brought about oxidative stress and inflammation, and then resulted in endothelial dysfunction, ultimately leading to the progression of DR. $^{34,35}$ Thus, the relationship between BMI and DR remains inconsistent. Abdominal obesity is considered as the risk for DR. ${ }^{36}$ However, Muhammad et al discovered that WC was inversely associated with the severity of $\mathrm{DR}^{32}$ The current research suggested that there was no relationship between BMI and DR. Nevertheless, the association of BMI and PNI level with DR was mutually independent. Therefore, for the high risk of DR with low PNI, neither high BMI nor low BMI could contribute to an increased risk of DR. The association between PNI and DR was also not affected by WC.

There are some potential explanations for the correlation between PNI and DR. Hypoalbuminemia often represents malnutrition and inflammation. With reduced protein synthesis, increased urine protein, and controlled diet in diabetic patients, serum albumin levels gradually decrease. Low albumin can cause increased permeability of the vessels in or around the optic disk, which leads to edema of optic disk, and ultimately result in compression of the central retinal artery and central retinal vein. Compression, in turn, results in high blood flow velocities in these vessels. ${ }^{37}$ Serum albumin is a primary constituent in the PNI formula, and thus can offer part of the explanation. $\mathrm{Wu}$ et al proposed that $\mathrm{T}$ cells participate in the development and progression of type 2 diabetic nephropathy (T2DN) ${ }^{38}$ Recent literatures have demonstrated that the neutrophil to lymphocyte ratio (NLR) was notably associated with the development and progression of DR and DN. ${ }^{39}$ Furthermore, a study discovered that the ratios of $\mathrm{CD} 4^{+} \mathrm{CD} 25^{\text {hi }}$ Tregs to Th17 or Th1 cells were declined in the diabetic individuals, accompanied by subsequent diabetic complications. ${ }^{11}$ DR and DN have 
certain parallelism in their development and progression. ${ }^{40}$ All these observations demonstrated that disordered $\mathrm{T}$ lymphocytes appeared to be associated with diabetic complications. Therefore, total lymphocyte count is another component of the PNI formula and thus could explain the increased DR risk in low PNI patients combined with hypoalbuminemia. Hence, taking our findings into account along with the above-mentioned explanations, PNI might be an effective and novel clinical marker for assessing the presence and severity of DR.

The strengths of this study design included a diagnosis of DR using fundus photograph with differing levels of $\mathrm{DR}$, comprehensive clinical and questionnaire protocols. And this study was the first to address the relationship between the PNI and the presence and severity of DR in patients with T2DM. Nevertheless, some potential limitations existed in the present study. First, any causal and effect on conclusions of PNI and the occurrence and development of DR could not be drawn because of the cross-sectional design. Second, the current study population was restricted to a single center, which limits the sample size. Third, due to PNI might be decreased in other conditions in T2DM except for DR and thus perhaps cause its low specificity, which limited the utility of PNI. The present findings should be confirmed and generalized in future researches based upon large sample-sizes with a control group of individuals with no diabetes. Meanwhile, longitudinal studies are required to confirm and generalize the findings, and to evaluate how PNI predicts the outcome of DR including visual impairment and blindness.

\section{Conclusion}

PNI was negatively and independently associated with the severity of DR. BMI and WC could not interfere with the association between PNI and severity of DR. It was suggested that PNI might be an effective, inexpensive and readily available biochemical indicator for assessing the presence and severity of DR in clinical practice. In addition, the immunonutrition-based notion could pave the way for a novel and supplementary solution for preventing DR or slowing the progression of DR.

\section{Acknowledgments}

This work was funded by the Natural Science Foundation of Zhejiang Province (Project Number: LY20H070003), Wenzhou Science \& Technology Bureau (Project
Number: 2020Y0164), and Youth Program of National Natural Science Foundation of China (81200576).

\section{Disclosure}

The authors report no conflicts of interest in this work.

\section{References}

1. Yau JW, Rogers SL, Kawasaki R, et al. Global prevalence and major risk factors of diabetic retinopathy. Diabetes Care. 2012;35 (3):556-564. doi:10.2337/dc11-1909

2. Prokofyeva E, Zrenner E. Epidemiology of major eye diseases leading to blindness in Europe: a literature review. Ophthalmic Res. 2012;47(4):171-188. doi:10.1159/000329603

3. Chung JO, Park SY, Chung DJ, Chung MY. Relationship between anemia, serum bilirubin concentrations, and diabetic retinopathy in individuals with type 2 diabetes. Medicine (Baltimore). 2019;98(43): e17693. doi:10.1097/MD.0000000000017693

4. Soro-Paavonen A, Forbes JM. Novel therapeutics for diabetic microand macrovascular complications. Curr Med Chem. 2006;13 (15):1777-1788. doi:10.2174/092986706777452515

5. Sharma Y, Saxena S, Mishra A, Saxena A, Natu SM. Nutrition for diabetic retinopathy: plummeting the inevitable threat of diabetic vision loss. Eur J Nutr. 2017;56(6):2013-2027. doi:10.1007/s00394017-1406-2

6. Elia M, Ceriello A, Laube H, Sinclair AJ, Engfer M, Stratton RJ. Enteral nutritional support and use of diabetes-specific formulas for patients with diabetes: a systematic review and meta-analysis. Diabetes Care. 2005;28(9):2267-2279. doi:10.2337/diacare.2 8.9.2267

7. Xie Y, Zhang H, Ye T, Ge S, Zhuo R, Zhu H. The geriatric nutritional risk index independently predicts mortality in diabetic foot ulcers patients undergoing amputations. $J$ Diabetes Res. 2017;2017:5797194. doi:10.1155/2017/5797194

8. Tang J, Kern TS. Inflammation in diabetic retinopathy. Prog Retin Eye Res. 2011;30(5):343-358. doi:10.1016/j.preteyeres.2011.05.002

9. Liu GX, Chen Y, Yang YX, et al. Pilot study of the mini nutritional assessment on predicting outcomes in older adults with type 2 diabetes. Geriatr Gerontol Int. 2017;17(12):2485-2492. doi:10.1 111 /ggi.13110

10. Wang H, Wang C, Wang L, et al. Orail downregulation impairs lymphocyte function in type 2 diabetes mellitus. Biochem Biophys Res Commun. 2018;500(2):384-390. doi:10.1016/j.bbrc.2018.04.083

11. Zeng C, Shi X, Zhang B, et al. The imbalance of Th17/Th1/Tregs in patients with type 2 diabetes: relationship with metabolic factors and complications. J Mol Med (Berl). 2012;90(2):175-186. doi:10.1007/ s00109-011-0816-5

12. Zhang C, Xiao C, Wang P, et al. The alteration of Th1/Th2/Th17/Treg paradigm in patients with type 2 diabetes mellitus: relationship with diabetic nephropathy. Hum Immunol. 2014;75(4):289-296. doi:10.1016/j.humimm.2014.02.007

13. Merlot AM, Kalinowski DS, Richardson DR. Unraveling the mysteries of serum albumin-more than just a serum protein. Front Physiol. 2014;12(5):299. doi:10.3389/fphys.2014.00299

14. Zhu Y, Cai X, Liu Y, et al. Serum albumin, but not bilirubin, is associated with diabetic chronic vascular complications in a Chinese type 2 diabetic population. Sci Rep. 2019;9:12086. doi:10.1038/ s41598-019-48486-6

15. Chinese Society of Endocrinology. Expert consensus on integrated management of type 2 diabetes mellitus complicated with obesity in China. Drug Eval. 2016;13(17):5-12.

16. Sasongko MB, Widyaputri F, Agni AN, et al. Prevalence of diabetic retinopathy and blindness in Indonesian adults with type 2 diabetes. Am J Ophthalmol. 2017;181:79-87. doi:10.1016/j.ajo.2017.06.019 
17. Low JR, Gan ATL, Fenwick EK, et al. Poor person and areal socioeconomic status is associated with increased diabetic retinopathy and visual impairment incidence and progression. Br J Ophthalmol. 2020; bjophthalmol-2020-316430. Doi:10.1136/bjophthalmol-2020-3 16430 .

18. Cederholm T, Barazzoni R, Austin P, et al. ESPEN guidelines on definitions and terminology of clinical nutrition. Clin Nutr. 2017;36 (1):49-64. doi:10.1016/j.clnu.2016.09.004

19. Saucillo DC, Gerriets VA, Sheng J, Rathmell JC, Maciver NJ. Leptin metabolically licenses $\mathrm{T}$ cells for activation to link nutrition and immunity. J Immunol. 2014;192(1):136-144. doi:10.4049/ jimmunol.1301158

20. Taylor AK, Cao W, Vora KP, et al. Protein energy malnutrition decreases immunity and increases susceptibility to influenza infection in mice. J Infect Dis. 2013;207(3):501-510. doi:10.1093/infdis/jis527

21. Najera O, Gonzalez C, Toledo G, Lopez L, Ortiz R. Flow cytometry study of lymphocyte subsets in malnourished and well-nourished children with bacterial infections. Clin Diagn Lab Immunol. 2004;11(3):577-580. doi:10.1128/CDLI.11.3.577-580.2004

22. Alwarawrah Y, Kiernan K, MacIver NJ. Changes in nutritional status impact immune cell metabolism and function. Front Immunol. 2018;9:1055. doi:10.3389/fimmu.2018.01055

23. Semeraro F, Cancarini A, dell'Omo R, Rezzola S, Romano MR, Costagliola C. Diabetic retinopathy: vascular and inflammatory disease. J Diabetes Res. 2015;2015:582060. doi:10.1155/2015/ 582060

24. Buzby GP, Mullen JL, Matthews DC, Hobbs CL, Rosato EF. Prognostic nutritional index in gastrointestinal surgery. Am J Surg. 1980;139(1):160-167. doi:10.1016/0002-9610(80)90246-9

25. Sun K, Chen S, Xu J, Li G, He Y. The prognostic significance of the prognostic nutritional index in cancer: a systematic review and meta-analysis. J Cancer Res Clin Oncol. 2014;140(9):1537-1549. doi:10.1007/s00432-014-1714-3

26. Cheng YL, Sung SH, Cheng HM, et al. Prognostic nutritional index and the risk of mortality in patients with acute heart failure. $J \mathrm{Am}$ Heart Assoc. 2017;6(6). doi:10.1161/JAHA.116.004876

27. Nakagomi A, Kohashi K, Morisawa T, et al. Nutritional status is associated with inflammation and predicts a poor outcome in patients with chronic heart failure. $J$ Atheroscler Thromb. 2016;23 (6):713-727. doi:10.5551/jat.31526

28. Tak BT, Cay S, Pamukcu HE, et al. Prognostic nutritional index as a novel marker for prediction of prognosis in patients with peripartum cardiomyopathy. Medicine (Baltimore). 2020;99(11):e19524. doi:10.1097/MD.0000000000019524

29. Correa-Rodriguez M, Pocovi-Gerardino G, Callejas-Rubio JL, et al. The prognostic nutritional index and nutritional risk index are associated with disease activity in patients with systemic lupus erythematosus. Nutrients. 2019;11(3):638. doi:10.3390/nu11030638
30. Sharma Y, Saxena S, Mishra A, Saxena A, Natu SM. Apolipoprotein A-I and B and subjective global assessment relationship can reflect lipid defects in diabetic retinopathy. Nutrition. 2017;33:70-75. doi:10.1016/j.nut.2016.08.012

31. Sharma Y, Saxena S, Saxena A, Mishra A, Natu SM. Interrelationship of elevated serum advanced glycation end-product levels and malnutrition (Subjective Global Assessment) scores with the severity of retinopathy in type II diabetes. Clin Nutr ESPEN. 2015;10(1):e42e48. doi:10.1016/j.clnesp.2014.12.002

32. Sasongko MB, Widyaputri F, Sulistyoningrum DC, et al. Estimated resting metabolic rate and body composition measures are strongly associated with diabetic retinopathy in Indonesian adults with type 2 diabetes. Diabetes Care. 2018;41(11):2377-2384. doi:10.2337/ dc18-1074

33. Man RE, Sabanayagam C, Chiang PP, et al. Differential association of generalized and abdominal obesity with diabetic retinopathy in Asian patients with type 2 diabetes. JAMA Ophthalmol. 2016;134 (3):251-257. doi:10.1001/jamaophthalmol.2015.5103

34. Kastelan S, Tomic M, Gverovic Antunica A, Ljubic S, Salopek Rabatic J, Karabatic M. Body mass index: a risk factor for retinopathy in type 2 diabetic patients. Mediators Inflamm. 2013;2013:436329. doi:10.1155/2013/436329

35. van Leiden HA, Dekker JM, Moll AC, et al. Blood pressure, lipids, and obesity are associated with retinopathy: the hoorn study. Diabetes Care. 2002;25(8):1320-1325. doi:10.2337/diacare.25.8.1320

36. Akazawa N, Okawa N, Hino T, Tsuji R, Tamura K, Moriyama H. Higher malnutrition risk is related to increased intramuscular adipose tissue of the quadriceps in older inpatients: a cross-sectional study. Clin Nutr. 2020;39(8):2586-2592. doi:10.1016/j.clnu.2019.11.028

37. Fujioka S, Karashima K, Nishikawa N, Saito Y. Optic disk manifestation in diabetic eyes with low serum albumin: late fluorescein staining and high blood flow velocities in the optic disk. Jpn J Ophthalmol. 2004;48(1):59-64. doi:10.1007/s10384-003-0005-3

38. Wu CC, Chen JS, Lu KC, et al. Aberrant cytokines/chemokines production correlate with proteinuria in patients with overt diabetic nephropathy. Clin Chim Acta. 2010;411(9-10):700-704. doi:10.1016/ j.cca.2010.01.036

39. Liu J, Liu X, Li Y, et al. The association of neutrophil to lymphocyte ratio, mean platelet volume, and platelet distribution width with diabetic retinopathy and nephropathy: a meta-analysis. Biosci Rep. 2018;38(3). doi:10.1042/BSR20180172

40. Pearce I, Simo R, Lovestam-Adrian M, Wong DT, Evans M. Association between diabetic eye disease and other complications of diabetes: implications for care. A systematic review. Diabetes Obes Metab. 2019;21(3):467-478. doi:10.1111/dom.13550

\section{Publish your work in this journal}

Diabetes, Metabolic Syndrome and Obesity: Targets and Therapy is an international, peer-reviewed open-access journal committed to the rapid publication of the latest laboratory and clinical findings in the fields of diabetes, metabolic syndrome and obesity research. Original research, review, case reports, hypothesis formation, expert opinion and commentaries are all considered for publication. The manuscript management system is completely online and includes a very quick and fair peer-review system, which is all easy to use. Visit http://www.dovepress.com/testimonials.php to read real quotes from published authors. 